\title{
Normalización de la información: la aportación de IraLIS
}

\author{
Por Tomàs Baiget, Josep-Manuel Rodríguez-Gairín, Fernanda Peset, Imma Subirats \\ y Antonia Ferrer-Sapena
}

\begin{abstract}
Resumen: La normalización de la información es imprescindible para transferirla, almacenarla y recuperarla. Afecta a todos los aspectos de su uso, y no sólo a los profesionales de su tratamiento. Este artículo aborda los aspectos más relevantes de la práctica profesional en este campo. Describe la solución implantada para nombres de autores españoles: IraLIS. Concluye insistiendo en la necesidad de que los autores científicos españoles se conciencien de que estas acciones mejoran la visibilidad de sus trabajos.
\end{abstract}

Palabras clave: Normalización, Estandarización, Nombres de autor, Autoridades de nombres personales

\section{Title: Information standardization: the IraL/S contribution}

Abstract: Standardization is essential for proper transfer, storage and recovery of information. Standardization affects all aspects of information usage, and not only for professionals in information processing. The most prominent aspects of professional practice in this field are adressed, including the IraLIS solution for citing Spanish authors with multiple surnames. There is a need for Spanish scientific authors to be aware that taking these actions will improve the visibility of their work in international citation resources.

Keywords: Standardization, Author names, Personal names citation standards, Personal name authorities

Baiget, Tomàs; Rodríguez-Gairín, Josep-Manuel; Peset, Fernanda; Subirats, Imma; Ferrer, Antonia. "Normalización de la información: la aportación de IraLIS". En: El profesional de la información, 2007, noviembre-diciembre, v. 16, n. 6, pp. 636-643.

DOI: 10.3145/epi.2007.nov.10

\section{Normalización de nombres}

LA NORMALIZACIÓN DE LA INFORMACIÓN es imprescindible para transferirla, $y$ afecta a todos los aspectos de la actividad humana, no sólo a los profesionales en el tratamiento de la información.

El trabajo de las entidades de normalización supera todas las barreras nacionales, como por ej., el establecimiento de los nombres de países o sus códigos de la ISO 3166 1 (1997) ${ }^{1}$, las Unidades Territoriales Estadísticas $^{2}$ (NUTS) utilizadas por la Comisión Europea, etc.

En el mundo de la información, según Herrero-Pascual (1999), el control de los nombres es una tarea clásica en el entorno bibliotecario, pues afecta directamente a la efica- cia de la recuperación de información. Además, ahora se rastrea un mundo infinito de información distribuida por internet y se extienden más las formas de trabajo colaborativas y virtuales (Ontalba, 2002 y 2006).

Tradicionalmente las bibliotecas han aceptado en sus ficheros de autoridad una forma del nombre personal o corporativo de un autor, haciendo referencia a él desde las formas no aceptadas. La IFLA promovió la edición de unas directrices para las entradas de autoridad y referencia, conocidas como GARE (Guidelines for authorities and reference entries), publicadas en 1984 (1993 en España) que abarcan los encabezamientos de autor personal, autor corporativo y títulos uniformes. Por otra parte, las directrices para los registros de autoridad y referencia de materias (Guidelines for subject authority and reference entries, GSARE), editadas en 1993 y traducidas en 1995, abarcan las entradas de materia y el lugar geográfico.

La segunda edición (2001) de las GARE (siglas desglosadas esta vez como Guidelines for authority records and references) —en las que intervino Assumpció Estivill como representante española-, fueron traducidas por la Dirección General del Libro, Archivos y Bibliotecas del Ministerio de Cultura, teniendo en cuenta las más recientes recomendaciones en cuanto a nivel mínimo para registros de autoridad (Minimum level authority record, $M L A R)$ que recoge un número, su lengua, la autoridad, entidad, 
referencias y notas), número normalizado (International standard authority data number, Isadn) y requisitos funcionales de los registros bibliográficos (FRBR).

Tillet (1996) nos recuerda que el Isadn es propuesto por la IFLA desde 1980, siendo uno de los éxitos de su programa Universal bibliographic control and international MARC (Ubcim). Hace unos años también los archiveros han editado las International standard archival authority record (for corporate bodies, persons and families) o Isaar (CPF).

Los objetivos básicos de las GARE y las GSARE fueron definir los elementos necesarios en los registros de autoridad, asignarles un orden y establecer su estructura. Seguían las directrices del programa Control bibliográfico universal $(C B U)$ en que las agencias nacionales se responsabilizaban de elaborar las listas de autoridades.

Pero actualmente la situación ha cambiado $\mathrm{y}$ se hace necesario un fichero virtual de autoridades a escala internacional que contemple diferentes formas de escritura pero un único acceso. Además los nuevos modelos conceptuales como Functional requirements for bibliographic records (FRBR) o Functional requirements and numbering of authority records (Franar) indican que el usuario lo que necesita es identificar sin ambigüedad, encontrar los conceptos, y acceder al item.

El control de la descripción ahorra tiempo y esfuerzo al usuario, mostrándole referencias de las entradas autorizadas. Ordena los registros bajo una sola forma, con indicación de las no aceptadas y permite generar automáticamente las variantes (orden directo, permutado, abreviado, otras lenguas y escrituras...). Para Tillet estos cambios vienen determinados por la aparición del web o más recientemente la Open archives initiative
$(O A I)$, que hacen posible una navegación por un universo casi infinito de recursos y precisan de un control persistente a la vez que interoperable entre los diversos formatos. Ya hoy la Oficina de desarrollo en red y estándares MARC de la Library of Congress ha elaborado un esquema de metadatos para autoridades en XML (Metadata authority description schema, MADS). Incluye los nombres de personas, de organizaciones, de congresos y eventos, de términos, de nombres geográficos..., para compartir en internet este tipo de información.

Estos nuevos escenarios, necesarios en el trabajo con autoridades, se concretan en diversos proyectos, algunos financiados por la Unión Europea, como Author, descrito por Danskin (1996). En él se realiza una equivalencia de los registros de autoridad de siete países al mismo formato de comunicación: Unimarc. También la OCLC a través de Connexion (antes CORC) busca la interoperabilidad, presentando los registros en MARC21 y Dublin core. Otros, como Linking and exploring authority files ( $L E A F)$, intentan enlazar los ficheros de autoridad usando los protocolos Z39.50 y OAI. Por su parte, los proyectos relacionados <indecs $>$ e Interparty promueven la cooperación entre bibliotecas, archivos y museos. Por último la Hong Kong Chinese authority for names (Hkcan), proporciona un fichero de autoridades compartido entre las bibliotecas de su consorcio, permitiendo la forma oriental, transcrita y simplificada.

El origen de estos esfuerzos puede verse en la sección Name authority cooperative program (NACO) del Program for cooperative cataloging (PCC) que nació en 1976 con un cariz casi exclusivamente anglosajón. Hoy día, no obstante, permite la participación de otras bibliotecas si son miembros del citado PCC. Byrum (2003) cifraba su oferta en dos millones de registros, con un aumento anual de 200.000 .

Para Tillet, aplicar un control de autoridades actualmente permite mayor precisión en la recuperación, algo casi imprescindible en un entorno web. Además, la estructura de los registros de autoridad permite navegar entre múltiples recursos de forma transparente para el usuario entre información diferente referida a una autoridad: nombre, explicación de variaciones, incoherencias, biografías, fuentes... (Weber, 2002, p. 5):

"It is widely accepted that the national and international sharing of authority information is a suitable means for reducing the costs of cataloguing work in libraries and archives as well as the costs for biographical research work undertaken in scientific projects”.

El nombre de un autor personal, a diferencia de los nombres de las instituciones $-\mathrm{O}$ autores corporativos- son independientes del idioma, especialmente si no necesitan transliteración. Al fin y al cabo son cadenas de caracteres unidas sin significado, y por tanto no son susceptibles de traducción o variación. Por ello resultan tan fáciles de recuperar de manera eficiente, al igual que la mayoría de nombres de empresas. Por ej., cuando alguien introduce en Google un nombre de persona o de una empresa suele obtener la información: de la empresa muy eficientemente porque usualmente tendrá un sitio web propio que aparece el primero en el ranking de resultados. Pero los autores, al figurar con variantes de nombres -nombre y apellido, apellidos y nombre, nombre desarrollado, con iniciales...- la labor de discriminación es ardua según el número de páginas que devuelva la consulta. El problema de los nombres es de singular importancia para el personal académico, que basa su reconocimiento profesional en su currículo personal e institucional. 


\section{Problema añadido para los autores españoles}

Las variaciones en las firmas cobran especial relevancia en España y en los países hispanoamericanos. A diferencia de los países anglosajones, portugueses, nórdicos y eslavos, usamos primero el apellido del padre y luego el de la madre, y muchas veces también un nombre de pila compuesto. El hecho de que una firma esté compuesta por muchos elementos contribuye, además, a que el autor firme sus trabajos de distintas formas. En España, por añadidura, también contamos con las variantes derivadas de las traducciones del nombre a los diferentes idiomas locales, adición de partículas, los apelativos o diminutivos... Los autores que firman con todo su nombre oficial (tal como figura en su DNI, pasaporte, etc.) se encuentran a menudo con la desagradable sorpresa de que sus trabajos publicados en revistas científicas aparecen referenciados de diferentes formas en los buscadores, en los depósitos OAI y en las bases de datos internacionales. Según como el productor del recurso haya interpretado su nombre aparecerán nuevas variantes, por lo que recopilar su bibliografía personal es en ocasiones muy difícil. En este contexto, la Fundación Española para la Ciencia y la Tecnología (Fecyt) publicó en enero de 2007 unas Recomendaciones (2007) para la firma de autores personales. El estudio de Ruiz, Delgado y Jiménez (2002) sobre las bases de datos ISI, Medline e $I M E$, permite identificar las formas en que los autores españoles firman y cómo aparecen reseñados.

Así, estas recomendaciones y el proyecto que ahora presentamos, IraLIS, mantienen una estrecha relación en sus principios. Existen, no obstante, dos diferencias. IraLIS es un banco de prueba para los autores de un área del conocimiento, biblioteconomía y documentación, muy sensible a este tipo de cuestiones de normalización, circulación, visibi- lidad, impacto... Por otra parte, tiene un alto componente tecnológico, desarrollado por Josep-Manuel Rodríguez-Gairín (Universitat de Barcelona), lo que permite obtener unos resultados directamente útiles para los autores científicos en las cuestiones apuntadas.

\section{La aportación de IraLIS}

IraLIS (International Registry for Authors in Library and Information Science) es un proyecto que surge en noviembre de 2006 a partir de las relaciones entre E-LIS (Eprints in library and information science), EXIT (Directorio de expertos en el tratamiento de la información) y la revista EPI (El profesional de la información).

Respectivamente E-LIS es un repositorio especializado en ciencias de la documentación, que cumple las normas OAI-PMH; EXIT es un directorio internacional con información sobre personas, instituciones y especialidades de trabajo; y por último, EPI es una revista española de nuestra profesión, la única que es indizada en ISI-Thomson y Scopus. Estos tres productos tienen en común su proyección internacional, su carácter documental, su distribución por internet, y el estar orientados al mundo de la gestión de la información.

http://www.iralis.org

http://eprints.rclis.org

http://www.directorioexit.info/

http://www.elprofesionaldelainformacion.com

Desde EXIT se recuperan los trabajos depositados en E-LIS a través de la interrogación de los nombres de los autores. De este vínculo surge la necesidad de trabajar en la normalización de los nombres de autoridades, algo que ya EPI había detectado al analizar la circulación de los trabajos de sus autores en las bases de datos internacionales.

El hecho de que todos estos productos sean complementarios entre sí y estén diseñados por personas vinculadas al mundo de la información determinó el comienzo de IraLIS para registrar, recomendar y recuperar las formas diversas en que un autor puede firmar. Su estrategia de normalización resulta ecléctica si la comparamos con el trabajo tradicional de catalogación de autoridades que hemos expuesto. Sin embargo, no deja de estar inmerso en este contexto, trabajando con autores personales y también corporativos -en tanto que calificadores de los autores personales-, como veremos más adelante. En definitiva es un desarrollo tecnológico que intenta superar los silencios que se producen en la recuperación de información sobre autores en internet. De sus resultados se beneficiarán en última instancia los proyectos mencionados y los autores que obtengan un iralis, es decir, el nombre registrado para su firma.

\section{Objetivos de IraLIS}

IraLIS se ha fijado unos objetivos a corto plazo, y otros a medio-largo plazo, pues las tareas a realizar requieren tanto acciones contundentes inmediatas (cuando están en la mano de sus promotores), como actuaciones más pausadas pero continuas de penetración en el entramado científico.

Se propone reducir la grave distorsión en la recopilación bibliográfica de los autores de tres formas:

1. Creando un registro de nombres de autores en biblioteconomía, documentación y archivística, que ayude a resolver las diferentes variantes. El registro incorporará tanto las que puede haber usado un autor, como las que haya interpretado el productor, agregador, buscador, etc., de las diversas fuentes de información.

2. Concienciando a los autores hispanos para que firmen sus trabajos siempre de la misma forma, pensando en cómo los referencia- 
rán las bases de datos internacionales, los archivos OAI y los robots de búsqueda. Sistemas que, como hecho consumado prácticamente irreversible, están bajo la influencia de la cultura y los hábitos ingleses (Science Citation Index, Scopus, Chemical Abstracts, Medline, Google Scholar, etc.).

3. Creando el sencillo formato de firma IraLIS, que permite ser interpretado adecuadamente y sin confusiones también por las fuentes de información de cultura anglosajona. A partir de esta forma aceptada (o iralizada) del nombre se pretende que un algoritmo de consulta en motores de búsqueda y harvesters permita recuperar todas las variantes.

Así, IraLIS no es únicamente un registro de la forma estandarizada del nombre, sino que se basa en la interoperabilidad de los sistemas y en la recuperación del nombre del autor desde diferentes bases de datos abiertas. Por ejemplo, IraLIS sabe contestar en XML a preguntas hechas en OpenURL, y el campo iralis del directorio EXIT muestra de forma dinámica los datos que están registrados en IraLIS. Esta funcionalidad permitirá igualmente que desde repositorios como E-LIS pueda validarse la introducción de autores mediante consultas directas a IraLIS usando tecnologías ajax.

\section{Funcionamiento}

El proyecto cuenta con un comité ejecutivo, formado por los miembros fundadores, que revisa la pertinencia de los iralis registrados para hacerlos visibles o no. Además, cuenta con un comité asesor que propone nuevas experiencias en este campo, sugiere mejoras o son consultados en caso de dudas. Actualmente forman parte de él representantes de organismos relacionados con la gestión y el estudio de la ciencia española, como son la $\mathrm{Fe}$ $c y t$, varias universidades, el Cindoc

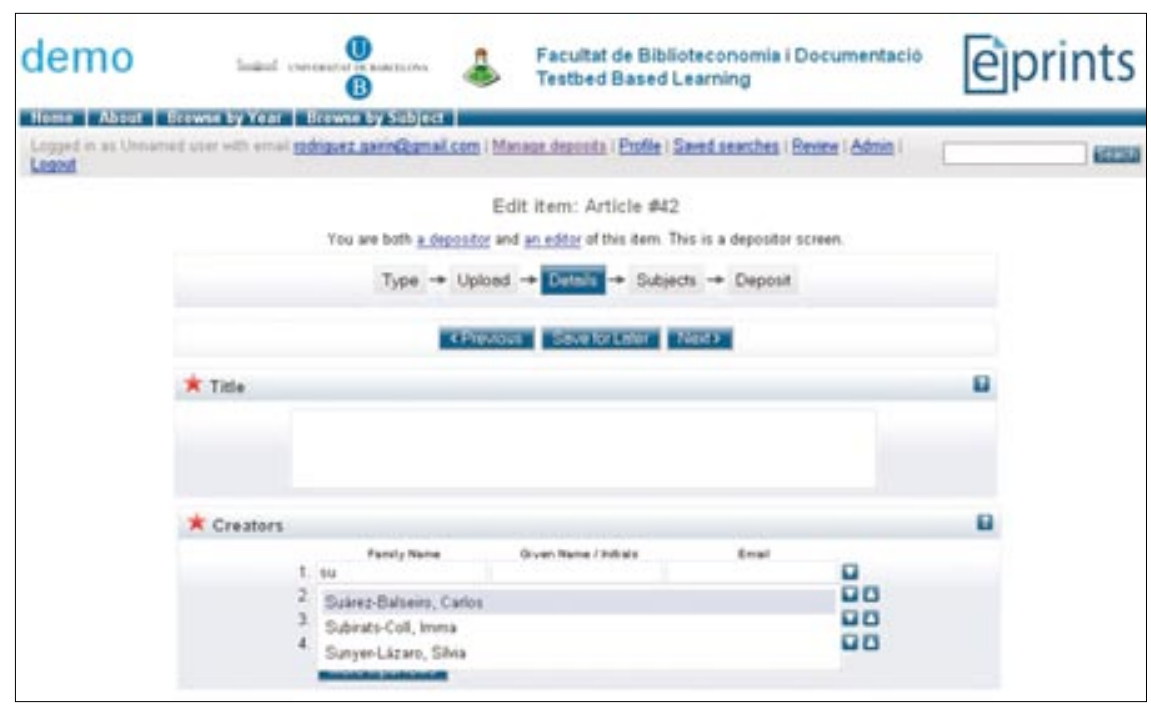

Figura 1. E-prints. Demo. http://eprints3.bd.ub.es/

o el Parc de Recerca Biomèdica de Barcelona ${ }^{3}$.

http://www.iralis.org/ $? q=$ node $/ 11$

La programación de IraLIS ha dado lugar a una base de datos o lista de autoridades, cuyos registros son generados por los propios autores rellenando un sencillo formulario.

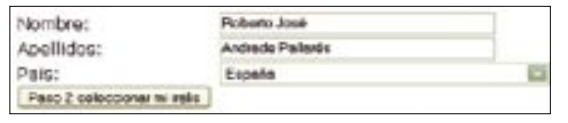

Figura 2. Register your IraLIS. http://www. iralis.org/?q=node $/ 8$

Una vez introducido el nombre completo, el sistema presenta una lista con las diferentes variantes que pueden constituir el iralis personal en forma inversa. Así, si un usuario introduce por ejemplo el nombre Roberto José Andrade Pallarés, el sistema le ofrece esta gama de opciones para que de entre ellas el usuario elija el iralis que prefiera.
A sugerencia de María Bordons (Cindoc) se omitieron algunas variantes de manera que se trate de una selección de posibilidades, no una lista exhaustiva:

Uno de estos formatos iraliscompatibles, que como se puede apreciar siempre están constituidos por 2 ristras de caracteres (con una eventual inicial intermedia opcional), debería ser el formato de firma fijo y universal de cualquier autor de trabajos científico-técnicos, susceptibles de ser recogidos por las bases de datos y los robots de búsqueda. En términos generales coincide con las Recomendaciones de la Fecyt, ya nombradas, usando guiones o combinando Nombre1 Nombre2 Apellido1 Apellido2.

Por último, IraLIS ofrece una recomendación de firma e información sucinta sobre el funcionamiento de las bases de datos bibliográficas. Una vez registrado, el sistema podrá utilizar tanto las variantes definidas por el autor como las au-

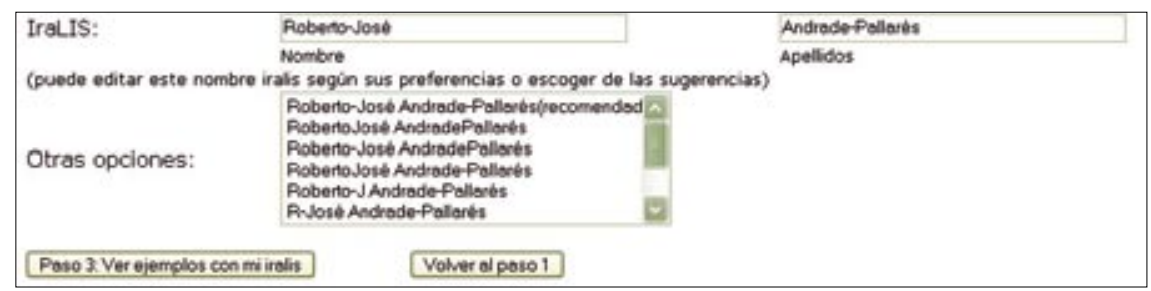

Figura 3. El sistema ofrece variantes normalizadas para que el interesado elija la que prefiere. La forma sugerida que se presenta en la primera línea puede editarse. 


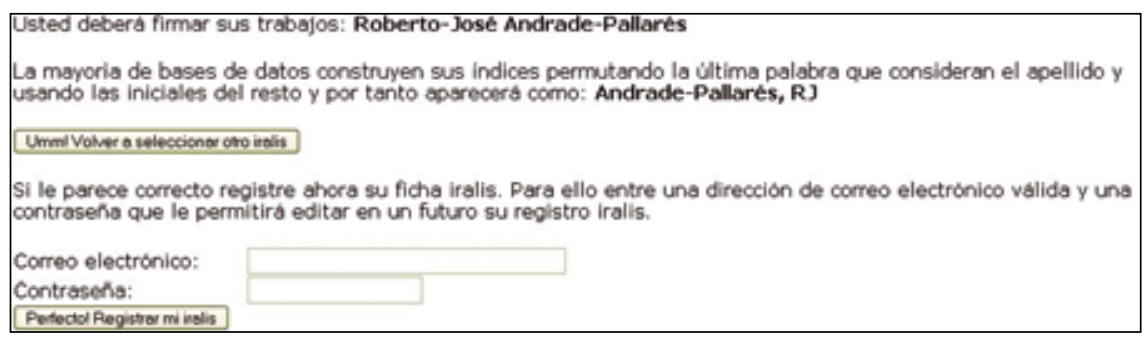

Figura 4. Registro definitivo del formato de firma elegido

togeneradas por IraLIS, junto con el correo electrónico del autor -a sugerencia de Isidro Aguillo, Cindoc- para la recuperación de información por internet.

Una vez registrado el iralis se puede ver en formato $M A D S$ y definir las variantes de nombre. Así también, presenta un enlace al $D i$ rectorio de Expertos en el Tratamiento de la Información (EXIT) que permite completar el registro con datos de tipo directorio.

Sin embargo puede ocurrir que en publicaciones oficiales no sea posible firmar con el iralis, o también puede ser que el autor no quiera usar ningún formato iralis. En estos casos el registro IraLIS, con todas las variantes, tanto las reales como las potenciales, seguirá siendo de gran utilidad cuando se quieran hacer búsquedas exhaustivas de las obras de un autor.

Estas reglas de validación del proyecto son claras: el nombre ha de estar compuesto por dos ristras que forman nombre y apellido. No aparecerán visibles en el proyecto las personas que deseen mantener otro tipo de firma. Aunque se permite el registro de cualquier formato de firma, el Comité Ejecutivo de IraLIS puede autorizar o no las formas introducidas según la regla de validación expuesta: dos únicas ristras de caracteres.

\section{Formato de los registros}

El registro de un autor está compuesto por:

ID_iralis: Un número

Fecha de alta: fecha de entrada en el registro

Nombre registrado: con un campo para los apellidos reales y un campo para el nombre real. Recoge la forma del nombre Iralis registrado: con un campo para los iralis-apellidos y un campo para el iralis-nombre. Recoge la forma aceptada.

Correo electrónico: usado por el autor

Password

Las variantes del nombre contienen los siguientes campos:

Id_iralis

Variante_nombre

Variante_apellidos
Cronología: con los campos

Desde y Hasta,

Comentarios

Cada autor posee un número iralis. Este identificador se autogenera con los datos que se poseen en la siguiente forma XXZZZ00000, donde $X X$ es el código del país, ZZZ es la especialidad (en previsión de la extensión del proyecto a otras disciplinas) y 00000 el número automático del registro de IraLIS. Así por ejemplo el ID de Tomàs Baiget es ESLIS00010.

Para normalizar los números de identificación los ficheros de autoridades que generan, por ejemplo, las bibliotecas nacionales se utiliza el International standard authority data number (Isadn). Pero existen otros números relacionados con grandes bases de datos como puede ser Scopus, de Elsevier, como la figura que mostramos:

O los de la Biblioteca del Con-

\begin{tabular}{|ll|}
\hline Baiget, $T$, \\
\hline Personal \\
\hline Name & \\
\hline Author ID & Baiget, $T$. \\
\hline
\end{tabular}

Figura 6. http://www.scopus.com/scopus/ author/profile.url?aid $=7801647379$ \&origin $=$ recordpage\&tx Gid $=$ W4bRv0OfFuhxm6jirb BXFS U\%3a20

greso de Washington (figura 7).

La forma aceptada con nombre $\mathbf{y}$ apellido. Es la forma del nombre de un autor, aceptada por él mismo. El autor podrá modificarla en cualquier momento que lo desee, almacenándose la forma antigua para poder seguir recuperándola. Siempre que cumpla la regla de validación aparecerá como aceptada $\mathrm{y}$ visible en el sistema. El formato iralis se concibe en forma directa, no anteponiendo apellido, aunque el sistema puede mostrarlo invertido según convenga, como por ejemplo para ofrecer las variantes, para
Figura 5. Ficha de autor con todas las variantes, históricas y actuales, de las firmas que ha usado o han interpretado quienes le han citado

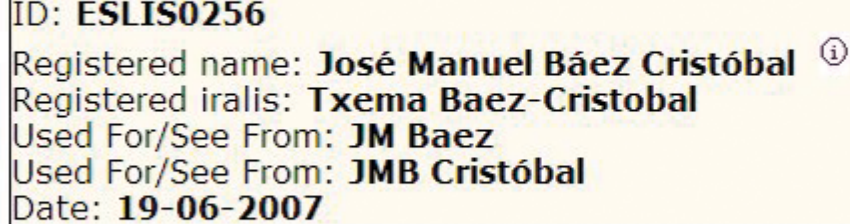

google " scholar ' oaister 


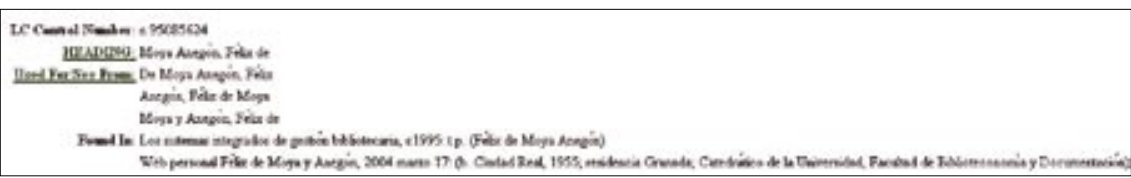

Figura 7. Library of Congress Authority.

http://authorities.loc.gov/cgi-bin/Pwebrecon.cgi?AuthRecID=1293991\&v2=1\&HC=1\&SEQ= $20070606112309 \& P I D=28270$

recuperarlas, para ordenarlas, para visualizarlas, etc.

Variantes con sus explicaciones. Además de las variantes propuestas automáticamente por el sistema, cada autor podrá introducir sus propias variantes, siempre que cumplan las reglas de validación de IraLIS. Así, por ejemplo, un autor puede tener un nombre determinado en el Registro Civil de su país, pero él puede decidir firmar con una variante iralis de su nombre: José, Xose, Josep, Joseph, Pepe, etc.

Las variaciones del nombre las puede introducir el mismo autor, indicando en qué momentos utilizó ese nombre y las razones. Pero también podrían ser volcadas desde los ficheros de autoridad, por ejemplo de la Biblioteca Nacional de España si su catálogo de autoridades se pudiera interrogar en XML. Consideramos que el porcentaje de auto- res que utilicen IraLIS y tengan entrada en la Biblioteca Nacional va a ser reducido, pues aparecen sólo los que cuentan con una publicación monográfica. Se mostraría en la ficha como una recopilación de las variantes.

Además el formato iralis es compatible con el esquema de descripción Metadata authority description schema (MADS), que transforma el MARC en xml, como muestra la siguiente figura 9 .

$\mathrm{Y}$ todo esto para conseguir recuperar la bibliografía de los autores exhaustivamente. Actualmente ya se encuentran implementados algoritmos de búsqueda de la forma aceptada y sus variantes en Google, Google Scholar y OAIster. Puede apreciarse en la imagen la diferencia de recuperación para un mismo autor:

En el caso de un motor de búsqueda recupera páginas web que donde aparece el nombre del autor. En el caso de bases de datos bibliográficas abiertas o harvesters como OAIster devuelve como respuesta referencias bibliográficas:

Google Scholar indiza los nombres de los autores en muchos casos por el último apellido (en nuestro ejemplo, Falgueras EA), por lo que no sería recuperable por el primero. Esto Google lo solventa porque indiza el texto completo normalmente el nombre correcto del autor es citado en el texto.

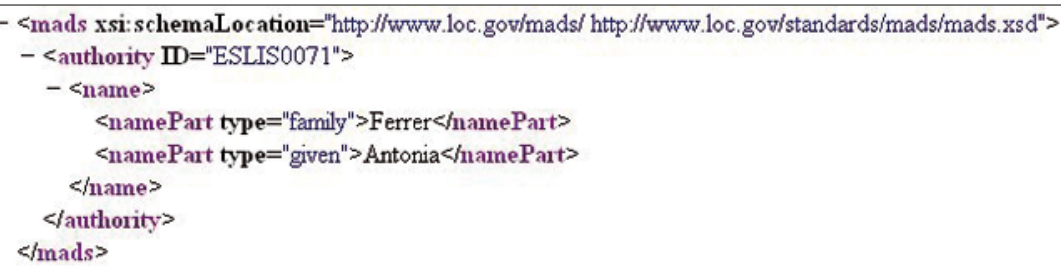

Figura 9. http://www.iralis.org/? $q=$ node $\% 2 F 8 \&$ paso $=10 \& l e t r a=F \& i d=71$

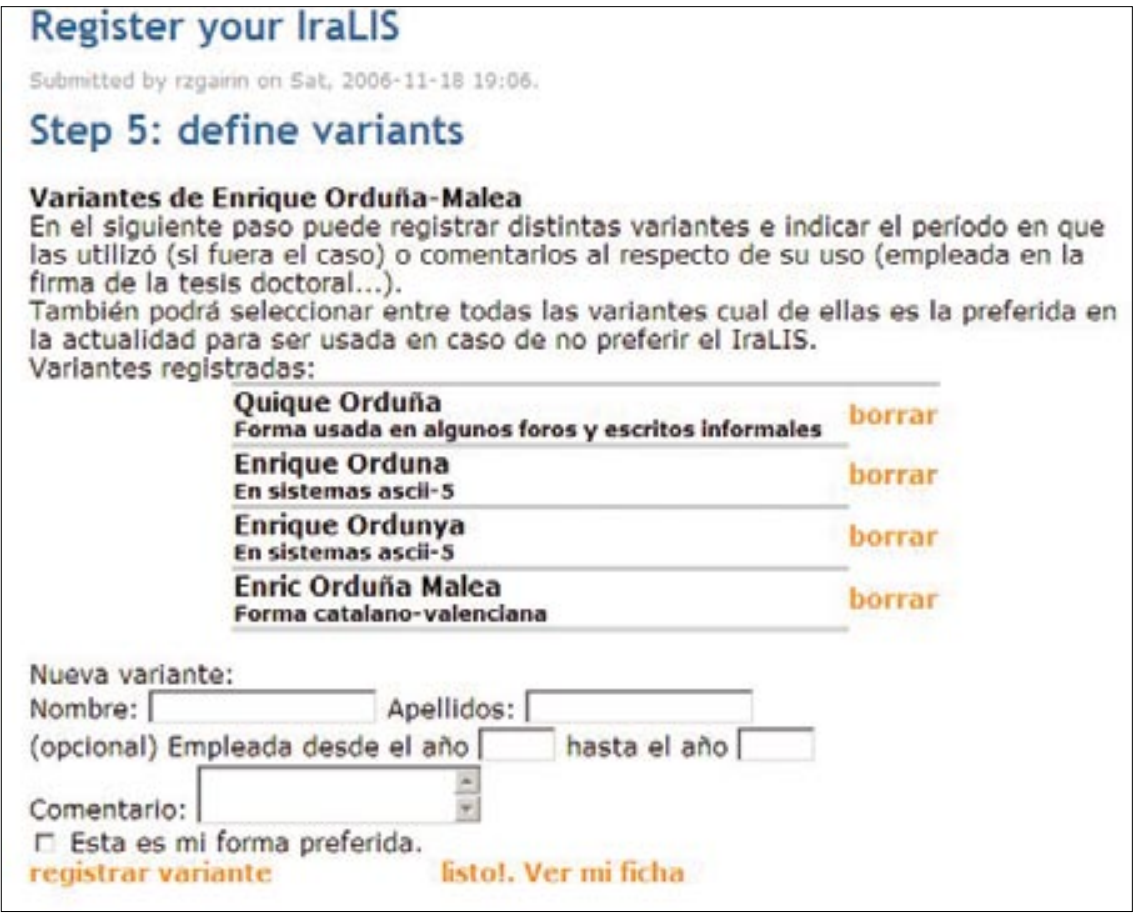

Figura 8. Form para introducir y definir variantes personales

\section{Conclusiones}

Hay que resaltar dos aspectos del proyecto IraLIS en cuanto a la normalización de la información y su posterior recuperación. En primer lugar, IraLIS es un sistema que ayuda a mentalizar a la gente para que firme siempre igual, que recomienda unas formas de firmar y que, en el futuro, recogerá todas las posibles variantes de las firmas de un autor, etc.

En segundo, "iralis" es un nombre de autor en un formato determinado, escogido por un autor de entre diferentes variantes, todas las cuales cumplen ser formatos iralis (dos bloques de caracteres). Por ejemplo tanto MF Peset como Fernanda Peset como Maria-Fernanda 


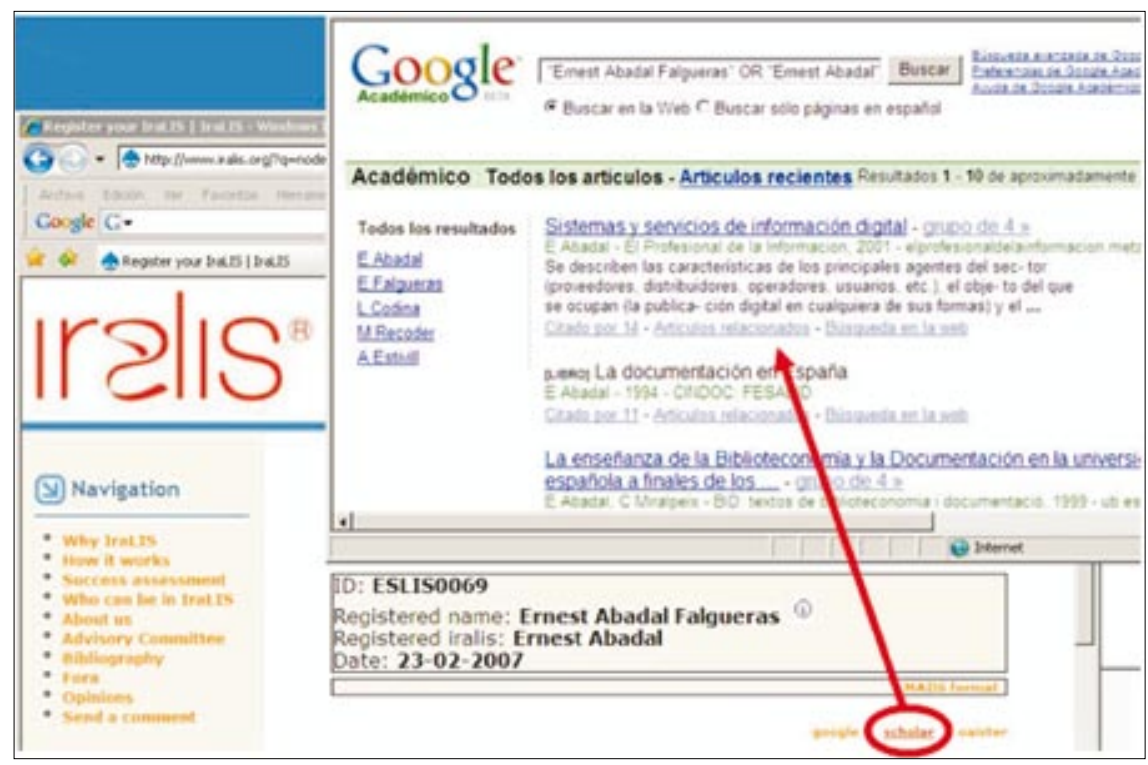

Figura 10. Búsqueda de un autor en Google Scholar (http://scholar.google.es/) a partir de su registro en IraLIS

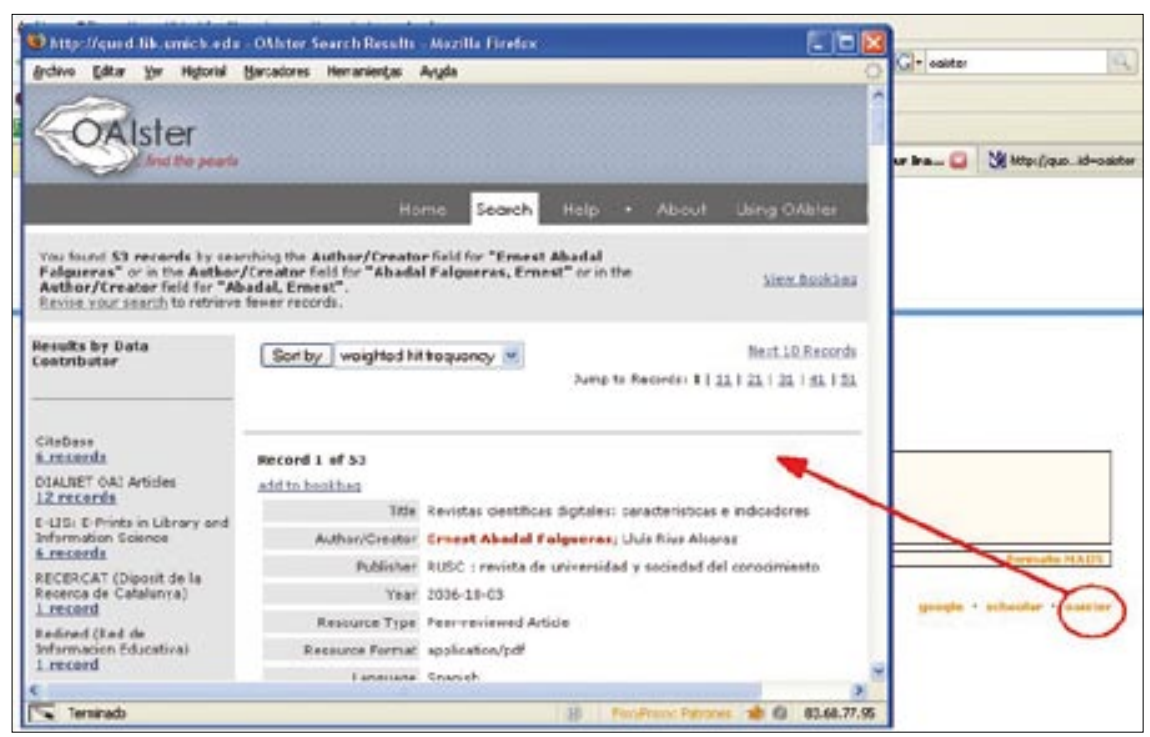

Figura 11. Búsqueda de un autor en OAlster (University of Michigan, Digital Library Production Service). http://www.oaister.org

Peset-Mancebo podrían serlo. El autor escogerá una de esas variantes y la pondrá como su iralis.

Este sistema tiene dos utilidades principales: permitirá la recuperación exhaustiva de bibliografías personales gracias a los registros del sistema IraLIS. A través de sus registros pueden recuperarse las variantes en cualquier base de datos. Estas variantes estarían constituidas por todas las formas no escogidas como iralis por el autor al registrarse en el sistema, el correo electrónico y todas las formas que desee introducir un autor, ya sea por que durante un tiempo firmara para extender su programación y la metodología de difusión a todos los campos de las ciencias en España.

\section{Notas}

1 Además a partir de la reunión en Santiago de Compostela en mayo de 2007 ISO/TC 46/WG 2 se consideró la posibilidad de trabajar en el código de nombre océanos y mares, países históricos, organizaciones internacionales...

2. Nomenclatura de las Unidades Territoriales Estadísticas utilizadas por la Unión Europea

3. Los actuales integrantes de Comité Asesor de IraLIS son: Isidro F. Aguillo (Cindoc, CSIC), Txema Báez (Fecyt), María Bordons (Cindoc, CSIC), Jordi Camí-Morell (Parc de Recerca Biomèdica de Barcelona), Emilio Delgado-López-Cózar (Universidad de Granada), Assumpció Estivill (Universitat de Barcelona), Isabel Gómez-Caridad (Cindoc, CSIC), Vicente P. Guerrero-Bote (Universidad de Extremadura), Félix de Moya-Anegón (Universidad de Granada) y Elías Sanz-Casado (Universidad Carlos III de Madrid)

\section{Bibliografía}

Bennett, Rick; Hengel-Dittrich, Christina; O'Neill, Edward T.; Tillett, Barbara B. "VIAF (Virtual international authority file): Linking die Deutsche Bibliothek and Library of Congress name authority files". En: World library and information congress: 72nd IFLA general conference and council 20-24 August 2006, Seoul, Korea. Consultado en: 26-10-2007.

http://www.ifla.org/IV/ifla72/papers/123-Bennett_trans-es.pdf http://www.ifla.org/IV/ifla72/ papers/123-Bennett-en.pdf

Buizza, Pino; Gerrini, Mauro. "Control de los puntos de acceso de autor y titulo". En: Principios de catalogación de IFLA: Pasos hacia un Código Internacional de Catalogación: Informe de la $1^{a}$ reunión IFLA de expertos sobre un Código internacional de catalogación, Frankfurt, 2003. Editado por Barbara B. Tillet, Renate Gömpel y Susanne Oehlschläger. Madrid: Subdirección General de Coordinación Bibliotecaria, 2005, pp. 121-151. Consultado en: 26-10-2007.

http://travesia.mcu.es/documentos/principios_ catalogacion_ifla.pdf

Byrum, John D. "NACO: a cooperative model for building and maintaining a shared name authority database". En: International conference authority control: definition and international experiences, Florence, Italy, February 10-12, 2003. Consultado en: 26-10-2007.

http://www.sba.unifi.it/ac/relazioni/byrum_eng. $p d f$

Callon, Michel; Courtial, Jean Pierre; Penan, Hervé. Cienciometría: la medición de la actividad científica, de la bibliometría a la vigilancia tecnológica. Gijón: Trea, 1995.

Danskin, Alan. "International standards in authority data control: costs and benefits". En: 62nd IFLA General Conference - Conference Proceedings - August 25-31, 1996. Paris: Inist, 1996. Consultado en: 22-06-2007.

http://www.ifla.org.sg/IV/ifla62/62-dana.htm 


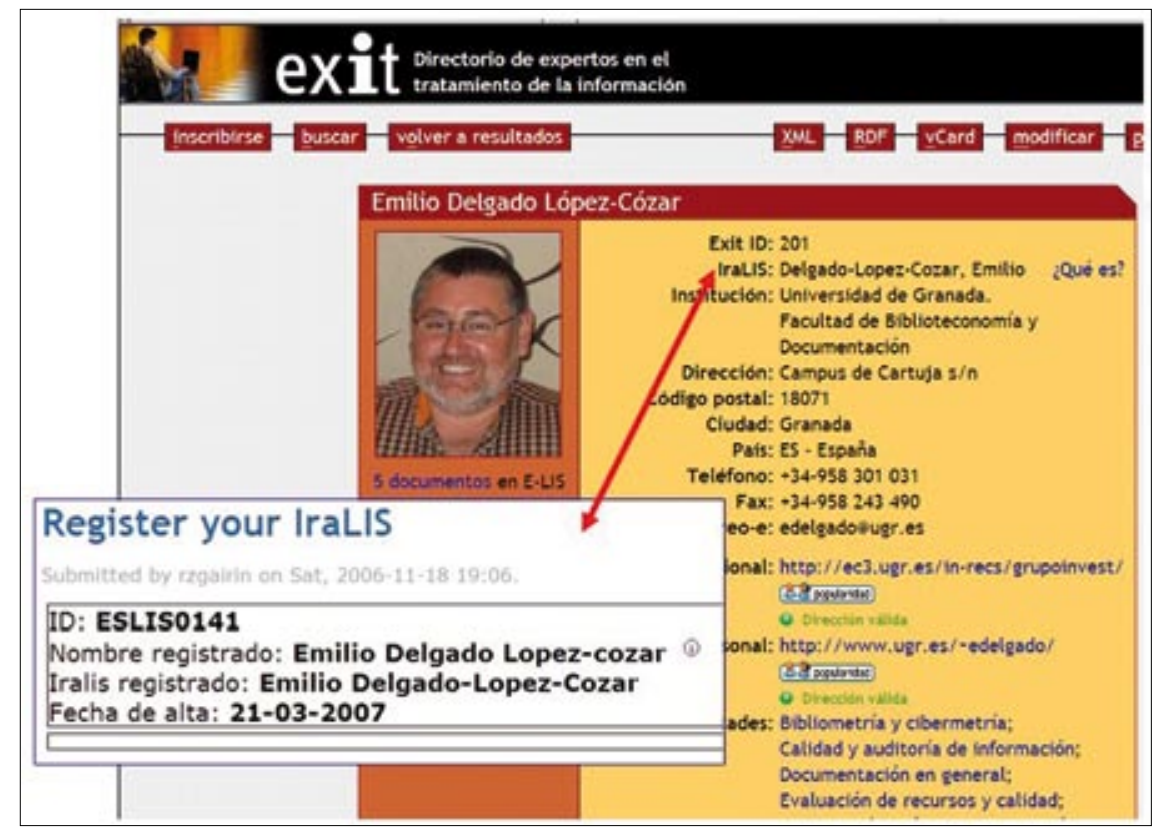

Figura 12. Entre las fichas de IraLIS y del Directorio EXIT existen enlaces en ambas direcciones

Estivill, Assumpció; Abadal, Ernest; Franganillo, Jorge; Gascón, Jesús; Rodríguez-Gairín, Josep-Manuel. "Uso de metadatos Dublin Core en la descripción y recuperación de artículos de revista digitales $=$ Use of Dublin Core metadata for describing and retrieving digital journals". En: Proceedings DC-2005 International conference on Dublin Core and metadata applications, 12-15 September. Madrid. Consultado en: 26-10-2007.

http://eprints.rclis.org/archive/00004792/

Estrategia nacional de ciencia y tecnología: Comisión Interministerial de Ciencia y Tecnología. Madrid: Fecyt, 2007.

Fecyt. Recomendaciones para la correcta identificación de las publicaciones científicas. Propuesta de manual de ayuda a los investigadores españoles para la normalización del nombre de autores e instituciones en las publicaciones científicas, 17 enero 2007.

http://www.accesowok.fecyt.es/recomendaciones_publicaciones.html

Formato Ibermarc para registros de autoridades. Madrid: Biblioteca Nacional, 1999.

Heijligers, Ton. “i’Entrada principal' en el futuro?" En: Principios de catalogación de IFLA. Pasos hacia un código internacional de catalogación: Informe de la $1^{a}$ reunión IFLA de expertos sobre un código internacional de catalogación, Frankfurt, 2003. Editado por Barbara B. Tillet, Renate Gömpel y Susanne Oehlschläger. Madrid: Subdirección general de coordinación bibliotecaria, 2005, pp. 153-158. Consultado en: 26-10-2007. http://travesia.mcu.es/documentos/ principios_catalogacion_ifla.pdf

Herrero-Pascual, Cristina. "El control de autoridades". En: Anales de documentación, 1999, $\mathrm{n}$. 2, pp. 121-136. Consultado en: 14-3-2007. http://www.um.es/fccd/anales/ad02/AD08-1999. $P D F$

IFLA. Directrices para los registros de autoridad y referencia de materia. Madrid: Anabad - Arco Libros, 1995.

IFLA. Directrices para los registros de autori- dad y referencia. Madrid: Anabad - Arco Libros, 1993.

IFLA. Directrices para registros de autoridad y referencias. Ministerio de Cultura, 2004. Consultado en: 26-10-2007. http://travesia.mcu.es/ documentos/directrices_autoridad.pdf

Indicadores bibliométricos de la actividad científica española (1990-2004). Madrid: Ministerio de Educación y ciencia, Fundación Española para la Ciencia y la Tecnología, 2007.

Informe SISE 2006: Sistema integral de seguimiento y evaluación, Observatorio permanente del sistema español de ciencia-tecnología-sociedad. Madrid: Fecyt, 2007.

ISO 3166-1 Codes for the representation of names of countries and their subdivisions. Part 1: Country codes. Ginebra: ISO, 1997.

LEAF (Linking and exploring authority files) Public progress LEAF's second year. Report 2. http://www.leaf-eu.org

Mandatory data elements for internationally shared resource authority records: Reports of the IFLA Ubcim working group on minimal level authority records and Isadn. Frankfurt: IFLA Ubcim Programme, 1998. Consultado en: 26-10-2007. http://www.ifla.org./VI/3/p1996-2/mlar.htm

Metadata authority description schema (MADS). Consultado en: 26-10-2007.

http://www.loc.gov/standards/mads/mads-outline.html

Ontalba-Ruipérez, José-Antonio. "Las comunidades virtuales académicas y científicas españolas: el caso de RedIris". En: El profesional de la información, 2002, sept.-oct., v. 11, n. 5, pp. 328-338.

Ontalba-Ruipérez, José-Antonio. "Las comunidades virtuales como herramientas para la socialización del conocimiento tácito". En: Documentación digital. Universidad Pompeu Fabra, 2006.

Peset, Fernanda; Ferrer, Antonia; Baiget, Tomàs; Rodríguez-Gairín, Josep-Manuel
"DSpace and the standardization of the information: names of Spanish authors". En: DSpace user group meeting 2007, FAO, Rome, Italy, October $17^{\text {th }}-19^{\text {th }}$.

Recomendaciones para la correcta identificación de las publicaciones científicas. Madrid: Fecyt, 2007. Consultado en: 10-03-2007. http://www. accesowok.fecyt.es/recomendaciones_publicaciones.html

Reglas de catalogación. Edición refundida y revisada. Madrid, Dirección General del Libro, Archivos y Bibliotecas, 1995.

Ruiz-Pérez, Rafael; Delgado López-Cózar, Emilio; Jiménez-Contreras, Evaristo. "Spanish personal names variations in national and international biomedical databases: implications for information retrieval and bibliometric studies". En: Journal of the medical library association, 2002, n. 90, pp. 411-430. Consultado en: 15-05-2007.

http://www.pubmedcentral.nih.gov/articlerender. fcgi? artid $=128958$

Tillett, Barbara B. "Authority control: State of the art and new perspectives". En: International conference authority control: definition and international experiences, Florence, Italy, February 10-12, 2003. Consultado en: 26-10-2007. http:// www.sba.unifi.it/ac/relazioni/tillett_eng.pdf

Tillett, Barbara B. "Un fichero de autoridades internacional virtual". En: Principios de catalogación de IFLA: Pasos hacia un Código internacional de catalogación: Informe de la $1^{a}$ reunión IFLA de expertos sobre un código internacional de catalogación, Frankfurt, 2003. Editado por Barbara B. Tillet, Renate Gömpel y Susanne Oehlschläger. Madrid: Subdirección general de coordinación bibliotecaria, 2005, pp.95-107. Consultado en: 26-10-2007. http://travesia.mcu. es/documentos/principios_catalogacion_ifla.pdf

Weber, Jutta. "Malvine, LEAF and Kalliope: Some co-operation models". En: Digital access to book trade archives (Papers of the 2001 Conference in The Hague). Leiden: Academic Press, 2002. pp. 49-68. Consultado en: 4-3-2007.

http://www.malvine.org/malvine/publications/ MALVINE_Weber_MLK.pdf

Tomàs Baiget, Institut d'Estadística de Catalunya (Idescat)

baiget@sarenet.es

\section{Josep-Manuel Rodríguez-Gairín, Universitat de Barcelona rodriguez.gairin@ub.edu}

Fernanda Peset, Universidad Politécnica de Valencia

mpesetm@upv.es

Imma Subirats, Food and Agriculture Organization (FAO), Roma imma.subirats@gmail.com

Antonia Ferrer-Sapena, Universidad Politécnica de Valencia anfersa@upv.es 


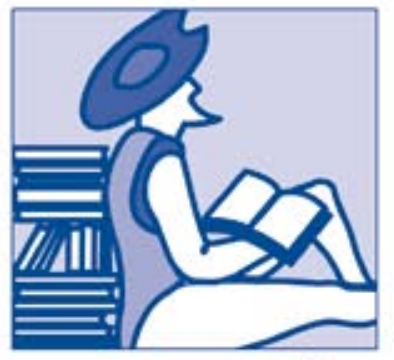

F U N A C I ÓN Alonso Quijano

\section{¿qué hacemos?}

Fomento de la lectura con menores hospitalizados.

Actividades de Formación.

Revista Mi Biblioteca.

Cooperación Internacional.

Recursos sobre lectura en la web.

\section{¿quieres colaborar?}

Hazte socio/a y recibirás estos dos libros de regalo*.

\footnotetext{
Cuota minima: $20 €$ al año.
}
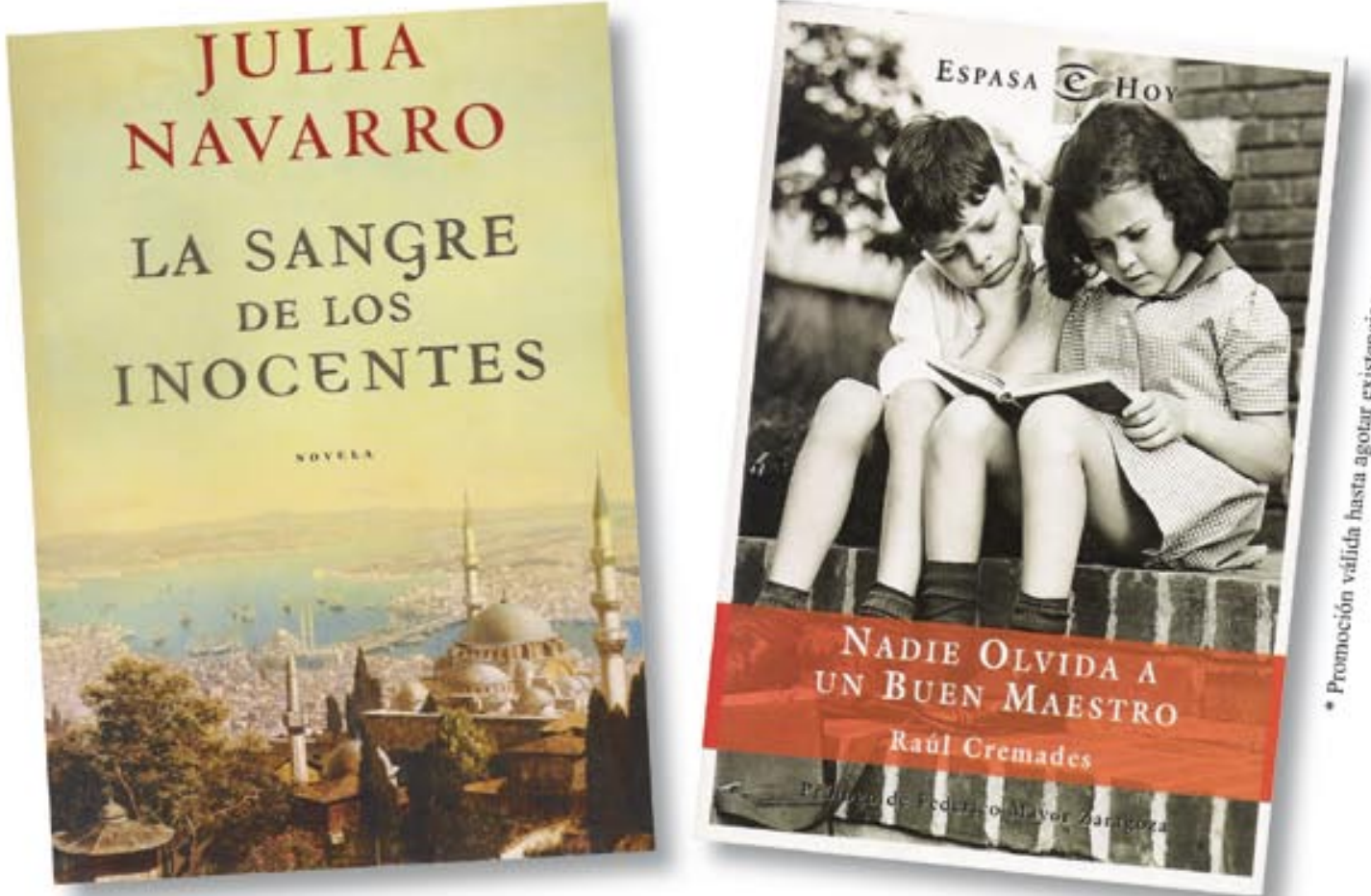

Puedes hacerlo por teléfono: 902362869 - 952235405 o a través de nuestra web: WWW.alonsoquijano.org

\section{Asóciate y disfruta de estas ventajas:}

- Regalo de un libro cada año como agradecimiento por la colaboración con la Fundación.

- Información sobre las actividades de la Fundación y participación en sorteos y promociones.

- Descuentos en suscripciones a revistas del sector.
- Descuentos en los cursos y otras actividades de formación organizadas por la Fundación.

- Regalo del Calendario de la Lectura que la Fundación publica cada año.

- Ventajas fiscales según la legislación vigente sobre mecenazgo. 